\title{
Radio Continuum Emission from the Magellanic Clouds.
}

\author{
U. KLEIN ${ }^{1}$, R.F. HAYNES ${ }^{2}$, R. WIELEBINSKI ${ }^{1}$, S.R. WAYTE ${ }^{3}$, J.I. HARNETT ${ }^{4}$, \\ E. BAJAJA ${ }^{5}$, J.D. MURRAY ${ }^{2}$, A.J. HUNT $^{2}$, R. WARK ${ }^{2}$, L. SCIACCA ${ }^{2}$, \\ U. R. BUCZILOWSKI ${ }^{1}$ \\ 1. Max-Planck-Institut für Radioastronomie. \\ 2. Australia Telescope National Facility, $\dagger$ \\ 3. Mount Stromlo and Siding Spring Observatories, \\ 4. University of Sydney, \\ 5. Instituto Argentino de Radioastronomia
}

ABSTRACT. New radio continuum surveys of the Large and the Small Magellanic Cloud have been completed recently at four frequencies, using the Parkes 64-m telescope. Here we shall discuss briefly the overall radio morphology of the Clouds at different frequencies and discuss the detected linear polarization. A preliminary integrated radio continuum spectrum of the Large Magellanic Cloud is also presented. Various aspects of future studies will be outlined.

\section{Introduction.}

Numerous studies of the Magellanic Clouds (MCs) have been carried out over a wide range of the electromagnetic spectrum. Owing to the proximity of the Clouds, very detailed measurements can be made with linear resolutions almost as good as those achievable in the Milky Way, but with all the advantages of the outsider's view. However, the large angular sizes involved pose problems for any investigation aimed at complete coverage of the Clouds. Enormous amounts of valuable telescope time are required for deep radio surveys of these galaxies.

After the first detection of the Large Magellanic Cloud at $85.5 \mathrm{MHz}$ by Mills and Little (1953), the MCs have been surveyed between $20 \mathrm{MHz}$ and $8.4 \mathrm{GHz}$. The low-frequency observations were aimed at the large-scale structure of the Clouds, while at higher frequencies, the emphasis was on the discrete source properties (see Mills and Turtle 1984, for a compilation). Another radio continuum survey was undertaken at $820 \mathrm{MHz}$ by Mills et al. (priv. comm.) at about the same time. These older surveys, however, did not provide complete coverages of the Clouds, and they suffered from poor signal-to-noise ratios. We decided therefore, to undertake more sensitive radio continuum surveys of the MCs at a number of frequencies to study their radio properties in detail. Conveniently, the Parkes 64-m telescope has been upgraded, and as a result of its new sensitive and very stable receiver systems, the new generation of surveys are an order of magnitude better than earlier ones. It was also convenient for us that our surveys were undertaken while the MCs were being thoroughly surveyed in other bands of the electromagnetic spectrum: in the far-infrared (FIR) (Rice et al. 1988; Schwering 1989; Schwering and Israel 1989), in X-rays (Helfand 1990), and in the CO line (Cohen et al. 1988). The Clouds have also been mapped in the radio continuum as part of the All Sky Surveys, namely at $408 \mathrm{MHz}$ by Haslam et al. $(1981 ; 1982)$, and at $2.3 \mathrm{GHz}$ by Mountfort et al. (1987).

Over the last two years, a complete multi-frequency radio continuum survey of the MCs has been accomplished using the Parkes 64-m telescope at $1.4 \mathrm{GHz}$ (Haynes et al. 1986), 2.4, 4.75, and $8.55 \mathrm{GHz}$ (Haynes et al. 1990). In this paper we present some more of the recently obtained radio continuum maps, and discuss the large-scale distribution of the radio emission of the MCs. Some aspects of the impact on various astrophysical problems are highlighted.

† The Australia Telescope National Facility is operated in association with the Division of Radiophysics by CSIRO

R. Haynes and D. Milne (eds.), The Magellanic Clouds, 31-35.

(C) 1991 IAU. Printed in the Netherlands. 


\section{New Radio Continuum Surveys.}

In Table 1 we have compiled the observational parameters of our radio continuum surveys of the Large and Small Magellanic Clouds (LMC and SMC, respectively). The 2.4-GHz survey does not have the same high quality as the others, owing to satellite interference. A new survey will be undertaken later this year, with the frequency centred at $2.5 \mathrm{GHz}$.

\section{TABLE 1 *}

\begin{tabular}{|c|c|c|c|c|c|c|c|c|c|}
\hline $\begin{array}{c}v \\
{[\mathrm{MHz}]}\end{array}$ & $\begin{array}{l}\text { Fiel } \\
{[0}\end{array}$ & $\begin{array}{l}\mathrm{d} \operatorname{siz} \\
\mathrm{x}\end{array}$ & & $\begin{array}{c}\text { \# of } \\
\text { coverages }\end{array}$ & $\begin{array}{c}\sigma \\
{[\mathrm{mJy}]}\end{array}$ & $\begin{array}{c}\text { HPBW } \\
{[\text { [] }}\end{array}$ & $\begin{array}{c}\mathrm{Tb} / \mathrm{S} \\
{[\mathrm{K} / \mathrm{Jy}]}\end{array}$ & $\begin{array}{l}\text { Obs. } \\
\text { date }\end{array}$ & \\
\hline 1401 & $\begin{array}{c}12 \\
6.5\end{array}$ & $\begin{array}{l}\mathbf{x} \\
\mathbf{x}\end{array}$ & $\begin{array}{l}12 \\
6.5\end{array}$ & $\begin{array}{l}4 \\
4\end{array}$ & 30 & 15.4 & 0.73 & Nov $_{4} 1984$ & $\begin{array}{l}\text { LMC } \\
\text { SMC }\end{array}$ \\
\hline$\underset{"}{2400}$ & $\begin{array}{c}12 \\
6.5\end{array}$ & & $\begin{array}{c}12 . \\
4.5\end{array}$ & $\begin{array}{l}3 \\
4\end{array}$ & $\begin{array}{l}50 \\
34\end{array}$ & 8.5 & 0.82 & Nov. 1987 & $\begin{array}{l}\text { LMC } \\
\text { SMC }\end{array}$ \\
\hline 47 & $\begin{array}{c}11 \\
6.5\end{array}$ & $\begin{array}{l}\mathbf{x} \\
\mathbf{x}\end{array}$ & $\begin{array}{l}10 \\
4.5\end{array}$ & $\begin{array}{l}3 \\
2\end{array}$ & $\begin{array}{l}8 \\
7\end{array}$ & 4.8 & 0.65 & Mar. 1988 & $\begin{array}{l}\text { LMC } \\
\text { SMC }\end{array}$ \\
\hline 8550 & $\begin{array}{l}7 \\
3.5\end{array}$ & $\hat{x}$ & $\begin{array}{l}6 \\
2.5\end{array}$ & $\begin{array}{l}2 \\
2\end{array}$ & $\begin{array}{l}17 \\
17\end{array}$ & 3.0 & 0.52 & Nov. 1988 & $\begin{array}{l}\text { LMC } \\
\text { SMC }\end{array}$ \\
\hline
\end{tabular}

In Figure 1 we show the total power maps of the LMC and the SMC at $8.55 \mathrm{GHz}$. These maps show that most of the emission at this frequency is correlated with HII regions as seen in $\mathrm{H} \alpha$ (Henize 1956; Davies et al. 1976) and in the FIR (Rice et al. 1988; Schwering 1989; Schwering and Israel 1989). Naturally, the diffuse nonthermal emission which dominates in these galaxies at lower frequencies (SMC: Loiseau et al. 1987; LMC: Klein et al. 1989) is much weaker at $8.55 \mathrm{GHz}$, and the thermal emission dominates the radio morphology of the Clouds at this frequency.

These maps of the LMC show the same overall asymmetry evident at lower frequencies. The dominant region in the radio continuum is the 30-Dor complex with its southern extensions. This region appears to host the largest gas reservoir as indicated from HI (Rohlfs et al. 1984) and CO (Cohen et al. 1988) maps. No excess radio emission has yet been detected in any of the surveys at the position of SN1987A.

\section{Linear Polarization.}

The maps at 2.4 and $4.75 \mathrm{GHz}$ exhibit strong linear polarization in the LMC south of 30 Dor. The apparent degree of polarization reaches $\sim 30 \%$ over a square degree $(900 \times 900 \mathrm{pc})$, and $\sim 10 \%$ is seen in a few other locations of the LMC. This indicates magnetic fields aligned over a large area. No significant polarization has been observed in the survey at $8.55 \mathrm{GHz}$ at the current sensitivity level. An important result is that the strongest polarization begins where the highest HI (Rohlfs et al. 1984) and CO (Cohen et al. 1988) column densities are seen. This may stress the role of magnetic fields for both the star-formation process itself and its preconditions.

In the SMC the situation is less conclusive. The linear polarization is very weak at all frequencies observed by us. We found that at $1.4 \mathrm{GHz}$ it reaches approximately $5 \%$ in some locations (Loiseau

* The 1.4 GHz maps and analysis of the LMC and SMC were published in Haynes et al. (1986) and in Klein et al. (1989). The $4.75 \mathrm{GHz}$ map of the LMC was published in Haynes et al. (1990) 
et al. 1987). It is also not much stronger at higher frequencies. The detected polarization at 2.4 and $4.75 \mathrm{GHz}$ is marginal, if present at all, and no polarization is seen at the sensitivity level of the 8.55 $\mathrm{GHz}$ measurements.

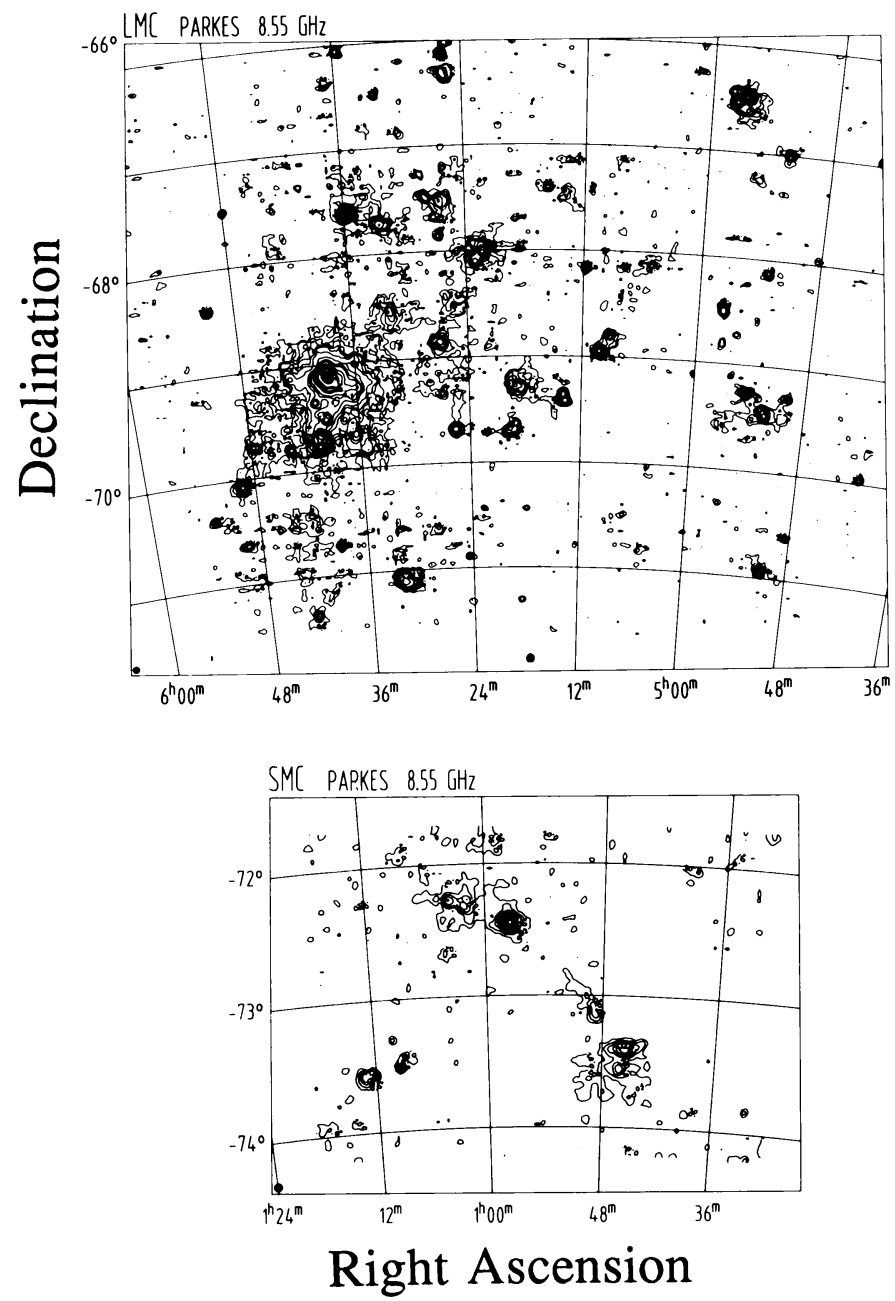

Fig 1

$8.55 \mathrm{GHz}$ survey of the Large Magellanic Cloud.

$4.75 \mathrm{GHz}$ survey of the Small Magellanic Cloud.

A likely interpretation of this may be twofold: at low frequencies, it could be caused by the large line-of-sight depth through the SMC, possibly coupled with a random field orientation along the line of sight (Loiseau et al. 1987); alternatively, at higher frequencies it could be the result of a dominance by thermal emission.

\section{Total Emission and Radio Spectrum.}

Accurate integrated flux densities for the LMC (Klein et al. 1989) and the SMC (Loiseau et al. 1987) have been derived for frequencies up to $2.3 \mathrm{GHz}$. We have computed preliminary integrated high-frequency flux densities for both objects, utilizing the new surveys between 2.4 and 8.55 GHz. In Figure 2 we show the integrated radio continuum spectrum of the LMC. Note that the flux 
densities at $2.4,4.75$, and $8.55 \mathrm{GHz}$ may still be inaccurate by several per cent, owing to inadequate consideration of (unrelated) background sources. At $8.55 \mathrm{GHz}$ the flux density is likely to be a lower limit because of incomplete coverage of the LMC field.

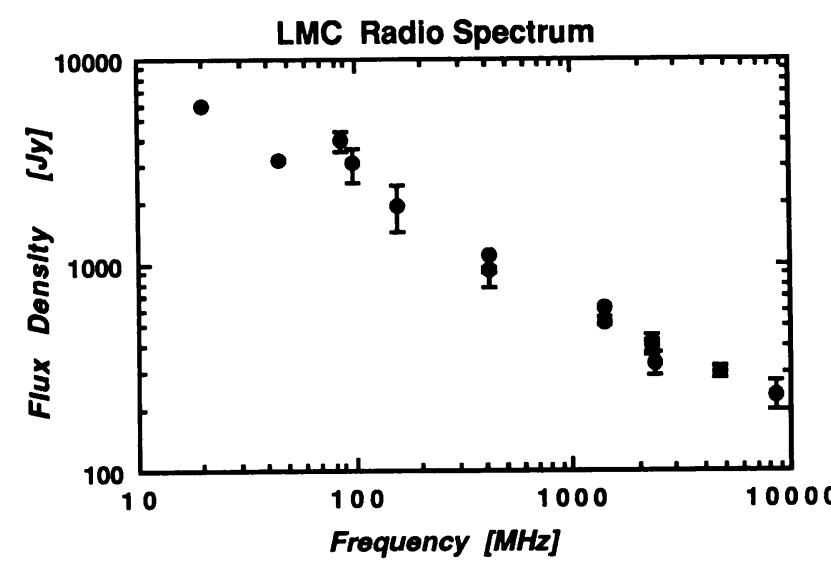

Fig 2

The low-frequency part of the spectrum in Figure 2 has $\alpha=-0.7\left(S_{V} \sim v^{\alpha}\right)$. We believe that the spectrum flattens significantly towards higher frequencies, indicating the prevalence of thermal emission. A least squares fit to the integrated flux density versus frequency spectrum indicates a mixture of a nonthermal spectrum $(\alpha=-0.7)$ and an optically thin thermal spectrum at the higher frequencies. For the SMC the situation is less conclusive at the moment: our preliminary flux densities at 2.4, 4.75 and $8.55 \mathrm{GHz}$ indicate a cutoff in the nonthermal spectrum at about $2-3$ GHz.

\section{Further aspects of this study.}

The radio continuum surveys which have been completed recently must be considered an important data base for numerous studies. First, they are very useful for detailed future studies (e.g. with the Australia Telescope). In particular, we are able to disentangle the varying contributions of thermal and nonthermal emission, down to a linear scale of $\sim 70 \mathrm{pc}$. This will support, for instance, the search for further supernova remnants. A comparison with X-ray observations (EINSTEIN, ROSAT) is therefore very worth while.

The detection of strong linear polarization in the LMC indicates regions of high magnetic field alignment over kpc scales. Along with this, and by separating the thermal and nonthermal components, we will be able to study cosmic ray generation and propagation in the LMC. This investigation is enhanced by incorporating the FIR (IRAS) data. IRAS data $(100 \mu$ and $60 \mu)$ may be used to some extent as an alias for the thermal radio emission (allowing consistency checks in the separation process). In addition, it provides a unique opportunity to scrutinize the well-known global radio-FIR correlation in galaxies (see for example Wunderlich and Klein 1988), down to scale sizes of $70 \mathrm{kpc}$.

Finally, the comparison of the radio continuum maps with the FIR (Rice et al. 1988; Schwering 1989; Schwering and Israel 1989), H $\alpha$ (Kennicutt and Hodge 1986), UV (Page and Carruthers 1981; Smith et al. 1987), CO (Cohen et al. 1988), and HI (Mathewson and Ford 1984; Rohlfs et al. 1984) will enable the most thorough study of star-forming processes in an external galaxy on scales between $\sim 70 \mathrm{pc}$ and kpcs. 


\section{References.}

Cohen, R.S., Dame, T.M., Garay, G., Montani, J., Rubio, M., Thaddeus, P.: (1988), Astrophys. J. 331, L95

Davies, R.D., Elliott, K.H., Meaburn, J.: (1976), Mem. R. Astron. Soc. 81, 89

Haslam, C.G.T., Klein, U, Salter, C.J., Stoffel, H., Wilson, W.E., Cleary, M.N., Cook, D.J., Thomasson, P.: (1981), Astron. Astrophys. 100, 209

Haslam, C.G.T., Salter, C.J., Stoffel, H., Wilson, W.E.: (1982), Astron. Astrophys. Suppl. 47, 1

Haynes, R.F., Klein, U., Wielebinski, R., Murray, J.D.: (1986), Astron. Astrophys. 159, 22

Haynes, R.F., Wayte, S.R., Harnett, J.I., Klein, U., Wielebinski, Buczilowski, U.R., Bajaja, E., Malin, D., Murray, J.D., Hunt, A.J., Wark, R., Sciacca, L.: (1990), in: Galactic and Intergalactic Magnetic Fields, IAU Symp. 140, (eds R. Beck, P.P. Kronberg \& R. Wielebinski), Kluwer, p. 205-209

Helfand, D.J.: (1990), this volume

Henize, K.G.: (1956), Astrophys. J. Suppl. 2, 315

Kennicutt, R.C., Hodge, P.W.: (1986), Astrophys. J. 306, 130

Klein, U., Wielebinski, R., Haynes, R.F., Malin, D.: (1989), Astron. Astrophys. 211, 280

Loiseau, N., Klein, U., Greybe, A., Wielebinski, R., Haynes, R.F.: (1987), Astron. Astrophys. 178,62

Mathewson, D.S., Ford, V.L.: (1984), in Structure and Evolution of the Magellanic Clouds, IAU Symp. 108, (eds. S. van den Bergh, \& K.S. de Boer), Reidel, Dordrecht, p.125

Mills, B.Y., Little, A.G.: (1953), Aust. J. Phys. 6, 272

Mills, B.Y., Turtle, A.J.: (1984), in Structure and Evolution of the Magellanic Clouds, IAU Symp. 108, (eds. S. van den Bergh, \& K.S. de Boer), Reidel, Dordrecht, p.283

Mountfort, P.I., Jonas, J.L., de Jager, G., Baart, E.E.: (1987), Mon. Not. R. Astron. Soc. 226, 917

Page, T., Carruthers, G.R.: (1981), Astrophys. J. 248, 906

Rice, W., Lonsdale, C.J., Soifer, B.T., Neugebauer, G., Kopan, E.L., Lawrence, A.L., de Jong, T., Habing., H.J.: (1988), Astrophys. J. Suppl. 68, 91

Rohlfs, K., Kreitschmann, J., Siegmann, B.C., Feitzinger, J.V.: (1984), Astron. Astrophys. 137, 343

Schwering, P.B.W.: (1989), Astron. Astrophys. Suppl. 79, 105

Schwering, P.B.W., Israel, F.P.: (1989), Astron. Astrophys. Suppl. 79, 79

Smith, A. M., Cornett, R.H., Hill, R. S.: (1987), Astrophys. J., 320, 609

Wunderlich, E., Klein, U.: (1988), Astron. Astrophys. 206, 47 